\title{
Functional Dyspepsia and Irritable Bowel Syndrome: Beyond Rome IV
}

\author{
Vincenzo Stanghellini \\ Policlinico S. Orsola-Malpighi, University of Bologna, Bologna, Italy
}

\begin{abstract}
Keywords
Functional gastrointestinal disorders - Rome criteria · Classification · Diagnosis · Treatment approach
\end{abstract}

\begin{abstract}
Background: The correct diagnosis of functional gastrointestinal disorders (FGIDs) is quite a challenge. The overlaps between syndromes can complicate the interpretation of clinical data. Summary: The incidence of functional digestive disorders and irritable bowel syndrome are still underestimated with the currently applied diagnostic tools, and the management of the seemingly elusive disease is not satisfactory. For this reason, the "Rome" criteria were created to provide a better understanding and classification of FGIDs. Key Messages: Rome diagnostic criteria and recommendations should be used in the design and performance of clinical studies in the field of functional dyspepsia and irritable bowel syndrome.

(c) 2018 The Author(s) Published by S. Karger AG, Basel
\end{abstract}

\section{Incidence of Functional Gastrointestinal Disorders}

Digestive symptoms are frequent in the general population, ranging between 10 and 30 per cent in industrialized countries, although with highly variable degrees of severity. In the majority of cases, traditional diagnostic

\begin{tabular}{ll}
\hline KARGER & $\begin{array}{l}\text { (c) } 2018 \text { The Author(s) } \\
\text { Published by S. Karger AG, Basel }\end{array}$ \\
E-Mail karger@karger.com & $\begin{array}{l}\text { This article is licensed under the Creative Commons Attribution- } \\
\text { NonCommercial-NoDerivatives 4.0 International License (CC BY- } \\
\text { NC-ND) (http://www.karger.com/Services/OpenAccessLicense). } \\
\text { Usage and distribution for commercial purposes as well as any dis- } \\
\text { tribution of modified material requires written permission. }\end{array}$
\end{tabular}

procedures fail to identify any organic, systemic or metabolic cause that is responsible for digestive function abnormalities and symptom perception, so that these conditions are classified as functional gastrointestinal disorders (FGIDs; Fig. 1). Although only about $25 \%$ of symptomatic individuals seek medical support, FGIDs represent $40 \%$ of diagnoses in gastroenterological settings [1], and they are one of the leading causes for referral to emergency care units [2], thus draining substantial amounts of healthcare resources. Functional dyspepsia (FD) and irritable bowel syndrome (IBS) are the most common FGIDs. The lack of non-invasive, cheap, readily available biomarkers for diagnosing FD and/or IBS makes doctors still uncertain about the clinical approach to these conditions, despite major advancements in the understanding of their pathophysiological mechanisms and relative therapeutic improvements in the last decades. Thus, a series of traditional diagnostic investigations are often performed and repeated despite negative findings, thus failing to provide appropriate explanations of the nature of symptoms and leaving patients frustrated and worried about their "strange" clinical problems. As a consequence, patients end up seeking new (often similarly useless) consultations $[3,4]$. The recently updated Rome IV criteria were designed not only to provide guidance for research studies in general and therapeutic trials in particular, but also to facilitate making a positive diagnosis of individual FGIDs, based on both, presence of characteristic symptoms and absence

Prof. Dr. Vincenzo Stanghellin

Policlinico S. Orsola-Malpighi, University of Bologna Via Albertoni 15

IT-40138 Bologna (Italy)

E-Mail v.stanghellini@unibo.it 

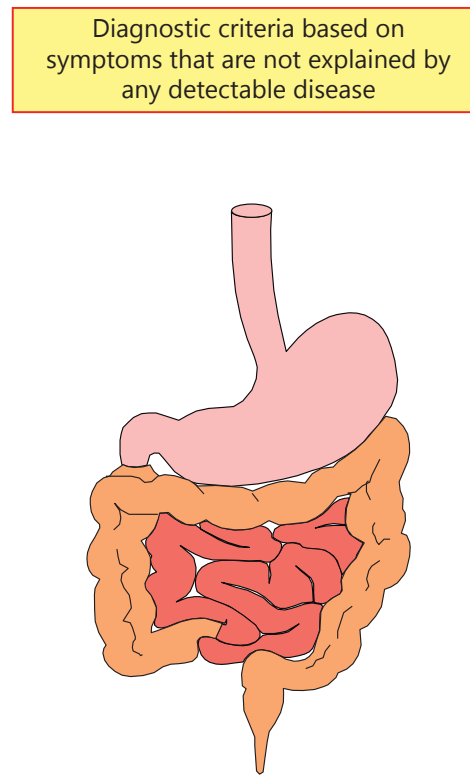

FGIDs in adults - 6 principal domains

- 27 disorders

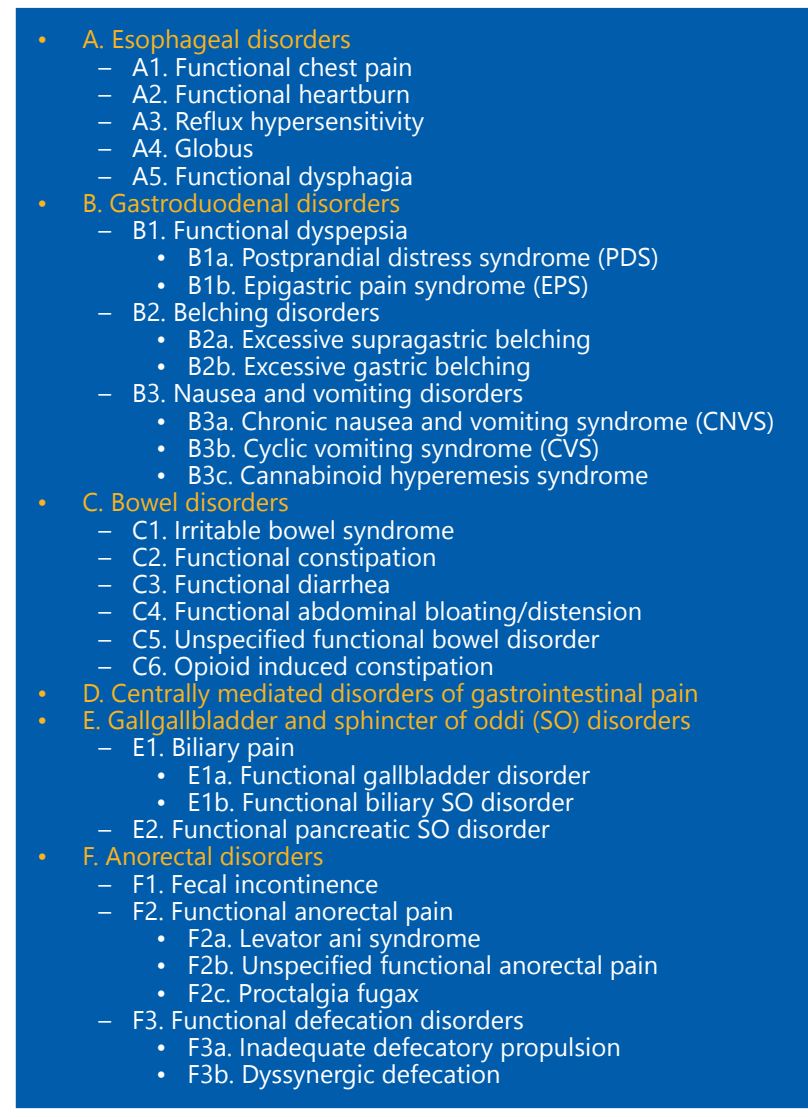

Fig. 1. Schematic representation of functional gastrointestinal disorders according to the Rome IV criteria [6].

of alarm features, and on objective findings from a limited number of standard diagnostic tests and investigations $[5,6]$.

\section{Rome Diagnostic Criteria and Differential Diagnosis}

The Rome IV criteria define dyspepsia as any combination of 4 symptoms: postprandial fullness, early satiety, epigastric pain, and epigastric burning that are severe enough to interfere with the usual activities and occur at least 3 days per week over the last 3 months with an onset of at least 6 months in advance [7]. After an accurate history taking and physical exam, in the absence of alarm symptoms and signs [6], patients are diagnosed as being affected by uninvestigated dyspepsia and can be treated empirically on the basis of their clinical manifestations. If the response is unsatisfactory or early relapses occur, a test and treat approach for Helicobacter pylori infection is recommended. Symp- toms of patients diagnosed with $H$. pylori associated dyspepsia are treated by $H$. pylori eradication. Patients in whom no identifiable explanation for the symptoms can be detected by traditional diagnostic procedures are diagnosed as being affected by FD. The umbrella term "FD" comprises patients from the following categories: (1) postprandial distress syndrome (PDS) that is characterized by meal-induced dyspeptic symptoms suggestive of a motility disturbance; (2) epigastric pain syndrome (EPS), that refers to epigastric pain or epigastric burning that do not necessarily occur after meal ingestion, may occur during fasting and can be even improved by meal ingestion, is reminiscent of the clinical features typical of a peptic ulcer and needs to be distinguished from gastro-esophageal reflux disease; and (3) overlapping PDS and EPS, characterized by mealinduced dyspeptic symptoms and epigastric pain or burning. The pathophysiology of FD is multifactorial and not fully understood. Gastroduodenal motor and sensory dysfunction as well as impaired mucosal integrity, low-grade 
immune activation, and dysregulation of the gut-brain axis have all been implicated [7]. The main motor abnormalities include delayed gastric empting and impaired distribution of gastric contents with inhibited gastric fundus accommodation and abnormally distended antrum, but they correlate only partially with symptoms' quality and severity. Gastric and duodenal hypersensitivities to distension, acid, lipids and other intraluminal stimuli can be found in subsets of dyspeptics. PDS patients are characterized by fasting and postprandial gastric hyper-mechanosensitivity, while EPS patients present decreased gastric compliance. Increased permeability of the mucosal barrier exposes the submucosal immune system to luminal noxious agents. Beyond $H$. pylori associated gastritis, duodenal eosinophilia has been reported in FD patients, suggesting potential new therapeutic approaches. Infections, stress, duodenal acid exposure, smoking and food allergy have all been implicated in the pathogenesis of duodenal mucosal inflammatory and permeability changes. Post-infectious dyspepsia has been reported, although it seems to be short-lived, compared to post-infectious IBS [8]. Psychosocial disorders may also play a role: anxiety, depression, neuroticism, as well as physical and emotional abuse and difficulty in coping with life events, are frequent among dyspeptic patients. A bidirectional relationship probably exists between gut and psyche: studies on the natural history of FGIDs suggest that patients affected by FD and IBS are particularly prone to develop psychological problems [9].

\section{Subforms of IBS}

The term IBS defines a syndrome characterized by recurrent abdominal pain in association with altered bowel habits (diarrhea, constipation, or both). According to the Rome IV criteria, symptom onset should occur at least 6 months before diagnosis, and symptoms should be present during the last 3 months [10]. Abdominal bloating and/ or distension or other forms of abdominal discomfort are frequently present, but are not included in the diagnostic definition of IBS. Frequency, duration, and severity of symptoms range from negligible to incapacitating, but are most often prompt modifications in the usual activities of affected individuals. IBS is subclassified into 4 categories; (1) IBS-C with predominant constipation (i.e., $>25 \%$ of bowel movements with Bristol stool form types 1 or 2 and $<25 \%$ of bowel movements with Bristol stool form types 6 or 7); (2) IBS-D with predominant diarrhea (i.e., $>25 \%$ of bowel movements with Bristol stool form types 6 or 7 and $<25 \%$ of bowel movements with Bristol stool form types 1 or 2); (3) IBS-M with mixed bowel habits (i.e., $>25 \%$ of bowel movements with Bristol stool form types 1 or 2 and $>25 \%$ of bowel movements with Bristol stool form types 6 or 7); and (4) unclassified IBS-U describing those patients who meet diagnostic criteria for IBS but whose bowel habits cannot be categorized into any of the three previous groups. The underlying pathophysiological mechanisms grossly resemble those described for FD, since an abnormal interplay among aggressive luminal contents, insufficient mucosal barrier, and excessive stimulation of submucosal immune system are involved in the stimulation of both intrinsic and extrinsic neural pathways, which in turn activate changes in digestive functions (including secretory and motor disorders) and symptom perception at the central nervous system level, respectively $[11,12]$. Moreover, as previously mentioned, abnormalities in the bi-directional interaction mechanisms between central and peripheral mechanisms are likely involved [9]. Genetic predisposition also seems to play a relevant role in polymorphisms detected in genes involved in immune activation, epithelial barrier, and host-microbiota interaction [13]. Enteroendocrine factors potentially involved include abnormal release of serotonin in IBS patients, irrespective of bowel habits, both during fasting and after meals [9]. Development of molecular techniques is promoting a rapid increase in our understanding of gut microbiota in several pathological conditions, including IBS. An increased Firmicutes/Bacteroides ratio and a decreased diversity of bacterial species seem to be of major relevance among the several abnormalities identified in subgroups of patients with IBS. Bacterial and viral pathogens responsible for infectious gastroenteritis represent the strongest known risk factor for the development of IBS (post-infectious IBS or PI-IBS). A correlation between gut dysbiosis and expression of several host gene pathways controlling cell junction integrity and inflammatory response has been detected in PI-IBS and IBS-D. As long suspected by patients and doctors alike, dietary factors may also be involved in the determination of the syndrome. Specifically, lactose and gluten sensitivities, as well as ingestion of excessive amounts of fermentable carbohydrates are thought to play relevant pathogenetic roles in the subsets of IBS patients. Increased mucosal permeability probably modulated by genetic factors, as well as by aggressive intraluminal contents and psychological or physical stressors expose the largest immune system of the human body, which is located in the gut submucosa, to antigenic activation, thus inducing the release of a cascade of inflammatory mediators, including histamine, cytokines, prostanoids, and proteases. A pivotal role is played by activated mast cells which are increased in the subsets of IBS
16

Dig Dis 2017;35:14-17

DOI: 10.1159/000485408
Stanghellini 
patients, regardless of their bowel habits [14]. The concentration of activated mast cells in proximity of nerve endings strongly correlates with the severity of abdominal pain reported by affected individuals. Enteric and extrinsic nerves stimulated by inflammatory mediators respond to relevant neuroplastic changes including increased expression of both neuronal growth factor and polymodal receptors, as well as neurite outgrowth and sprouting. Taken together, these data suggest that an abnormal mucosal milieu induced by immune activation plays a role in the pathophysiology of IBS, inducing long-lasting neuroplastic changes that are responsible for gut secretory, motor and sensory dysfunctions that characterize the disease.

\section{Conclusions}

Despite the substantial understanding of the underlying pathophysiological mechanisms and the high prevalence of IBS, as one of the most frequent diagnoses made by general practitioners and gastroenterologists, its management remains challenging in both settings. In the absence of specific biomarkers to objectively confirm a diagnosis of IBS, the Rome IV guidelines indicate that the diagnosis of IBS requires: (1) a thorough history taking including severity of the conditions and possible alarm features and triggering factors such as food intolerances, psychosocial conditioning factors, etc.; (2) a physical exam to evaluate possible palpable masses, abdominal distension, skin abnormalities, etc.; and (3) limited traditional diagnostic tests capable of distinguishing IBS from other pathological conditions which may present with similar symptoms, such as celiac disease, IBD, and microscopic colitis [10]. Key issues in the management of IBS patients are all those factors that are necessary to establish a strong doctor-patient relationship. It is mandatory that the patient is reassured about the "reality" of its condition and educated to somewhat understand the "micro-organic" (i.e. cellular/molecular) mechanisms that are responsible for the determination, onset, fluctuations, and chronicity of the symptoms. Determining the predominant disorder in bowel habits is important, since it may provide guidance for treatment approaches, although IBD-C and IBS-D often overlap and share, at least to a certain extent, common underlying mechanisms.

For a better comparability of clinical reports, the Rome criteria should be used in clinical trials testing the efficacy of medicinal products aimed at the treatment of FD and IBS.

\section{Disclosure Statement}

Vincenzo Stanghellini is a member of the advisory board and/ or speaker for AlfaSigma, Allergan, Angelini, Bayer, Malesci, Takeda, and receives scientific support from AlfaSigma and Allergan.

\section{References}

1 Russo MW, Gaynes BN, Drossman DA: A national survey of practice patterns of gastroenterologists with comparison to the past two decades. J Clin Gastroenterol 1999;29:339-343.

2 Caporale N, Morselli-Labate AM, Nardi E, Cogliandro R, Cavazza M, Stanghellini V: Acute abdominal pain in the emergency department of a university hospital in Italy. United European Gastroenterol J 2016;4:297-304.

3 Collins J, Farrall E, Turnbull DA, Hetzel DJ, Holtmann G, Andrews JM: Do we know what patients want? The doctor-patient communication gap in functional gastrointestinal disorders. Clin Gastroenterol Hepatol 2009;7: 1252-1254.e1-e2.

4 Stewart M, Brown JB, Donner A, McWhinney IR, Oates J, Weston WW, Jordan J: The impact of patient-centered care on outcomes. J Fam Pract 2000;49:796-804.

5 Drossman DA: The functional gastrointestinal disorders and the Rome III process. Gastroenterology 2006;130:1377-1390.

6 Drossman DA: Functional gastrointestinal disorders: history, pathophysiology, clinical features and Rome IV. Gastroenterology 2016;150:1262-1279.
7 Stanghellini V, Chan FK, Hasler WL, Malagelada JR, Suzuki H, Tack J, Talley NJ: Gastroduodenal disorders. Gastroenterology 2016; 150:1380-1392.

8 Cremon C, Stanghellini V, Pallotti F, Fogacci E, Bellacosa L, Morselli-Labate AM, Paccapelo A, Di Nardo G, Cogliandro RF, De Giorgio R, Corinaldesi R, Barbara G: Salmonella gastroenteritis during childhood is a risk factor for irritable bowel syndrome in adulthood. Gastroenterology 2014;147:69-77.

9 Koloski NA, Jones M, Talley NJ: Evidence that independent gut-to-brain and brainto-gut pathways operate in the irritable bowel syndrome and functional dyspepsia: a 1-year population-based prospective study. Aliment Pharmacol Ther 2016;44: 592-600.

10 Lacy BE, Mearin F, Chang L, Chey WD, Lembo AJ, Simren M, Spiller R: Bowel disorders. Gastroenterology 2016;150:1393-1407.

11 Barbara G, De Giorgio R, Stanghellini V, Cremon C, Salvioli B, Corinaldesi R: New pathophysiological mechanisms in irritable bowel syndrome. Aliment Pharmacol Ther 2004; 20(suppl 2):1-9.
12 Barbara G, Stanghellini V: Biomarkers in IBS when will they replace symptoms for diagnosis and management? Gut 2009;58:1571-1575.

13 Ek WE, Reznichenko A, Ripke S, Niesler B, Zucchelli M, Rivera NV, Schmidt PT, Pedersen NL, Magnusson P, Talley NJ, Holliday EG, Houghton L, Gazouli M, Karamanolis G, Rappold G, Burwinkel B, Surowy H, Rafter J, Assadi G, Li L, Papadaki E, Gambaccini D, Marchi S, Colucci R, Blandizzi C, Barbaro R, Karling P, Walter S, Ohlsson B, Tornblom H, Bresso F, Andreasson A, Dlugosz A, Simren M, Agreus L, Lindberg G, Boeckxstaens G, Bellini M, Stanghellini V, Barbara G, Daly MJ, Camilleri M, Wouters MM, D'Amato M: Exploring the genetics of irritable bowel syndrome: a GWA study in the general population and replication in multinational casecontrol cohorts. Gut 2015;64:1774-1782.

14 Barbara G, Stanghellini V, De Giorgio R, Cremon C, Cottrell GS, Santini D, Pasquinelli G, Morselli-Labate AM, Grady EF, Bunnett NW, Collins SM, Corinaldesi R: Activated mast cells in proximity to colonic nerves correlate with abdominal pain in irritable bowel syndrome. Gastroenterology 2004;126:693-702. 